\title{
Prevalence and correlates of diabetes and metabolic syndrome in a rural indigenous community in Baja California, Mexico
}

\author{
Lorena S. Pacheco ${ }^{1,3^{*}}$ (D), David A. Hernández-Ontiveros ${ }^{2}$, Esmeralda Iniguez-Stevens ${ }^{3}$, Stephanie Brodine ${ }^{3}$, \\ Richard S. Garfein ${ }^{1,4}$, Margarita Santibañez ${ }^{3}$ and Miguel A. Fraga ${ }^{2}$
}

\begin{abstract}
Background: Diabetes is a leading cause of morbidity and mortality in Mexico and understudied among indigenous populations. This study aimed to determine the prevalence and identify correlates of Type 2 diabetes mellitus (Type 2 DM) and metabolic syndrome (MetS) in a rural, indigenous community in Northwestern Mexico.

Methods: A cross-sectional study was conducted in the community of San Quintin, Baja California, Mexico, among a sample of households. A total of 275 participants ( $\geq 18$ years old) underwent a questionnaire, physical examination, and serologic test. Prevalence and adjusted odds ratio (AOR), using logistic regression modeling, were estimated with 95\% confidence intervals (95\% Cl).

Results: The prevalence of Type 2 DM and MetS was 21.8 and 53.1\%, respectively. Mean \pm standard deviation (SD) age and body mass index of study participants was $35.8 \pm 13.0$ years and $28.7 \pm 5.6 \mathrm{~kg} / \mathrm{m}^{2}$, respectively. Participants were $75 \%$ female and $60.7 \%$ self-identified as indigenous. Thirty-seven percent of adults had high blood pressure. After controlling for age, higher educational attainment had a protective effect on Type $2 \mathrm{DM}$ (AOR $=0.39 ; 95 \% \mathrm{Cl} 0.20,0.77$ ). Additionally, the presence of MetS was associated with being female ( $\mathrm{OOR}=2.27 ; 95 \% \mathrm{Cl} 1.23,4.14)$ and having lower educational attainment ( $\mathrm{AOR}=0.62 ; 95 \% \mathrm{Cl} 0.37,0.94)$.

Conclusions: The prevalence of Type $2 \mathrm{DM}$ and MetS was high in this rural and indigenous population, and education was shown to play a critical role. These findings support the need for community-inclusive health-promoting interventions in rural communities.
\end{abstract}

Keywords: Type 2 diabetes mellitus, Metabolic syndrome, Prevalence, Rural population, Indigenous groups, Public health

\section{Background}

Type 2 diabetes mellitus (Type $2 \mathrm{DM}$ ) and metabolic syndrome (MetS) are global public health problems $[1,2]$. Type $2 \mathrm{DM}$ is a chronic disease with hyperglycemia as a hallmark [2]. MetS refers to a cluster of factors including central obesity, hyperglycemia, hypertriglyceridemia, low plasma high-density lipoprotein (HDL)-cholesterol and hypertension, where the presence of at least three of these factors indicates MetS [3]. Entities including the National Cholesterol Education Program Adult Treatment Panel III

\footnotetext{
* Correspondence: 12pachec@ucsd.edu

${ }^{1}$ Department of Family Medicine and Public Health, School of Medicine, University of California, San Diego, La Jolla, CA, USA

${ }^{3}$ School of Public Health, San Diego State University, San Diego, CA, USA Full list of author information is available at the end of the article
}

(ATP III), the World Health Organisation and the International Diabetes Federation (IDF), have developed MetS diagnosis guidelines [4-6]. MetS components underlie cardiometabolic diseases increasing the risk of developing diabetes and cardiovascular disease (CVD), and even mortality $[7,8]$. It has been shown that there is a five-fold increased risk in Type $2 \mathrm{DM}$ development among those with MetS [9].

The prevalence of these two conditions continues to increase in parallel. Diabetes prevalence has almost doubled in the last 35 years globally, and it is estimated that between 10 and $40 \%$ of the world's adult population has MetS $[9,10]$. In Latin America, there is a MetS prevalence (weighted mean) of $24.9 \%$ (range: $18.8-$ 43.3\%) [11]. According to data from the 2006 Mexican 
National Health and Nutrition Survey (ENSANUT 2006) the prevalence of MetS could be as high as $49.8 \%$ in the overall adult population [12]. Mexico has the fifth highest number of individuals with diabetes, after China, India, the United States and Brazil [9], with diabetes accounting for the leading cause of morbidity [13]. The ENSANUTMedio Camino 2016, a mid-point national survey, determined the diabetes prevalence to be $9.4 \%$ nationally, and 9.6\% in rural northern areas of Mexico [14].

There has been limited research on Type $2 \mathrm{DM}$ and cardiovascular risk factors in Mexican indigenous populations, and even less emphasis on MetS. A 2007 cross-sectional study found a prevalence of Type $2 \mathrm{DM}$ in an indigenous population in the central valley of Mexico of 4.4\% [15]. Another study found a prevalence of Type 2 DM among indigenous groups in neighboring Southern Mexican states of $8.7 \%$ among Zapotec and $6.9 \%$ among Mixe groups [16]. Data from a 2008 study in a migrant Mixtec population in rural Northwestern Mexico, found the prevalence of MetS was 41.1\%, while that of Type 2 DM was 26.2\% [17].

The aim of this study was to determine the prevalence and correlates of Type $2 \mathrm{DM}$ and MetS in adults in a rural, indigenous community in Baja California, Mexico, while addressing sex differences for burden of disease. The current study tackled some of the limitations of previous work in the same community, where the prevalence of Type $2 \mathrm{DM}$ and MetS was determined using a convenience sample and medical records of self-identified Mixtec adults [17].

\section{Participants and methods}

\section{Study design and protocol}

This cross-sectional community-wide study was conducted in a rural community in San Quintin, Baja California, Mexico (2013-2014), led by Viaje Interinstitucional de Integración Docente, Asistencial y de Investigación (VIIDAI), a binational inter-institutional program involving two universities in the United States (University of California San Diego and San Diego State University [SDSU]) and one in Mexico (Universidad Autónoma de Baja California ([UABC]). VIIDAI faculty and students operate a weekend-long clinic twice a year, where primary care services are offered to underserved community residents. San Quintin is an agriculture-centered municipality located about $300 \mathrm{~km}$ south of the United States-Mexico border on the west coast of the Baja California Peninsula. Since agriculture plays a key role in the town's economy, it attracts farmers from South Mexico, primarily Oaxaca, in search of year-round work; the majority self-identify as indigenous. According to the latest surveillance data from Mexico's Indigenous Infrastructure Program, there were 3805 persons living in the rural community we studied, of which $51 \%$ were adults [18].
Formative research, six months prior to the study start date, involved geographical information system (GIS) mapping, supplemented by fieldwork to confirm all edifices, to produce a map from which to sample residences for the current study. The sampling frame consisted of an enumerated list of 779 residences successfully identified as occupied households, of which 340 sampling units (i.e., homes) were randomly pre-selected via simple random sample selection. This approach was taken to maximize study efficiency, using study personnel wisely while capturing a representative sample of homes from all sections of the community.

The study protocol consisted of visiting each pre-selected household and requesting an adult to be screened for eligibility. Only one adult per household was allowed to participate in the study therefore a die (highest number rolled) was used to select the participant. Eligibility criteria included residing in the neighborhood for $\geq 6$ months, age $\geq 18$ years old, and agreement to participate in the study. Before the comprehensive examination, written informed consent in Spanish was obtained from all participants. The examination took place in mobile units stationed within two blocks of participants' residence and involved an interviewer-administered questionnaire, anthropometric measurements, and blood sample. Following the examination, participants attended a nutritional counseling session and received $\$ 100.00$ pesos (Peso Mexicano) as supplementary nutritional support. The research team was trained on the study protocol and followed a culturally-sensitive approach.

The study procedures were approved by the Bioethics Committee of the Facultad de Medicina y Psicología of UABC and by the Institutional Review Board of SDSU's Human Research Protection Program.

\section{Socio-demographic and lifestyle questionnaire}

Participants were interviewed in private by trained and native Spanish-speaking surveyors, following a standardised script and validated 45-item questionnaire to obtain socio-demographic and lifestyle data [19]. Socio-demographic questions included age, sex, occupation, years living in San Quintin, literacy, and number of school years completed. Lifestyle questions addressed physical activity, smoking habits, and alcohol consumption. Physical activity was assessed by a composite score of four variables, each graded from one (least activity) to four (most activity), for a maximum score of sixteen points. Variables ranked from least to most activity, included: (1) main mode of transportation: automobile, motorcycle, bicycle, walking; (2) physical activity intensity at work: sitting, standing and walking, carrying light load/climbing stairs, heavy work; (3) frequency of recreational activity (e.g. sport): none or rarely, 2-3 times/month, 1-2 times/ 
week, and $>3$ times/week); and (4) hours per week of watching television: $>13 \mathrm{~h}, 8-12 \mathrm{~h}, 4-7 \mathrm{~h}$ and $<3 \mathrm{~h}$.

\section{Anthropometric and clinical measurements}

Height, weight, waist circumference, and blood pressure, were measured by trained research assistants following Mexican public health and clinical guidelines [20, 21]. Height and weight were determined to the nearest 0.1 $\mathrm{cm}$ and $0.1 \mathrm{~kg}$, respectively, using a mobile combined scale and stadiometer (Healthteam, GA, USA). Waist circumference was measured to the nearest $0.1 \mathrm{~cm}$ using a standard, non-stretch measuring tape (Gulik, Lafayette Instrument $\mathrm{Co}$, IN, USA). Body mass index (BMI) was calculated as weight in kilograms divided by height in meters squared.

Blood pressure was obtained using a new digital blood pressure monitor (Omron 7 series Plus) from participants in a seated position after resting for $10 \mathrm{~min}$, with arms relaxed and palms facing upward. Two readings were taken $5 \mathrm{~min}$ apart and the average of the two readings was recorded. If the difference between the first and the second reading was $\geq 10 \mathrm{mmHg}$ for systolic blood pressure (SBP) and/or $\geq 6 \mathrm{mmHg}$ for diastolic blood pressure (DBP), then a third measurement was done, and the average of all three measurements was calculated.

Anthropometry and blood pressure training was led by a study physician, certified dietitian-nutritionist and project researcher.

\section{Biochemical assays}

A non-fasting venous blood sample of $5 \mathrm{cc}$ was obtained from each participant. Assays included triglycerides, total cholesterol, and both low-density lipoprotein (LDL)- and HDL-cholesterol. The CardioChek PA (Polymer Technology Systems Inc., IN, USA) determined the lipid panel with $40 \mu \mathrm{l}$ of whole blood.

Glycated hemoglobin (HbA1c) was measured using the rapid diagnostic and point of care NycoCard HbA1c Reader II (Alere, MA, USA), utilising $5 \mu \mathrm{l}$ of blood from a finger stick sample. Both devices were calibrated every 10 tests.

\section{Outcomes measures}

Type $2 \mathrm{DM}$ was defined as $\mathrm{HbA} 1 \mathrm{c} \geq 6.5 \%$ according to American Diabetes Association (ADA) diagnosis criteria [22]. Participants that met the ADA's diagnosis criteria for Type $2 \mathrm{DM}$ were counseled by a physician and their test results were explained. A nutritional counseling session with a registered dietitian-nutritionist followed. Additionally, a sealed envelope that included a copy of their laboratory results and a clinical summary was provided to each participant, to take to their primary care physician for follow-up treatment and management.

MetS was defined using the IDF criteria [6]. This included participants with ethnic-specific waist circumference cut- offs of $\geq 90 \mathrm{~cm}$ in men and $\geq 80 \mathrm{~cm}$ in women. Additionally, participants had to meet at least two of the following to criteria: 1) elevated serum triglycerides $(\geq 1.7 \mathrm{mmol} / \mathrm{L}) ; 2)$ low HDL-cholesterol levels $(<1.03 \mathrm{mmol} / \mathrm{L}$ in men and $<1.20 \mathrm{mmol} / \mathrm{L}$ in women); 3) elevated SBP $\geq 130 \mathrm{mmHg}$ or DBP $\geq 85 \mathrm{mmHg}$; and 4) elevated fasting serum glucose levels, $(\geq 5.6 \mathrm{mmol} / \mathrm{L})$, or previously diagnosed diabetes.

\section{Statistical analysis}

Sample characteristics were expressed as mean \pm standard deviation (SD) for continuous variables, and frequencies and percentages for categorical variables. Prevalence of Type $2 \mathrm{DM}$ and MetS, and cardiovascular risk factors were stratified by sex. Student's t tests, and Pearson's Chi-square tests were used to assess the associations between each factor and binary outcome variables. For analyses, completed school years was dichotomised $(<6$ years vs $\geq 6$ years) based on mean of five schooling years, and income was dichotomised ( $<\$ 3200$ vs $\geq \$ 3200)$ based on median earnings.

Separate multivariable logistic regression analyses were performed to identify factors independently associated with Type 2 DM and MetS. Backward stepwise elimination was used to remove non-significant variables until only statistically significant variables remained in the final model. Unadjusted and adjusted odds ratios and corresponding 95\% confidence intervals were computed using simple and multivariable logistic regression analysis. Goodness of fit for the models was determined with the Hesmer-Lemeshow test. A $p<0.05$ denoted statistical significance. SAS version 9.2 (SAS Institute) was used for all statistical analyses.

\section{Results}

A total of 294 adults participated, corresponding to an $86 \%$ response rate. Ineligibility of participants and/or unresponsiveness explain the remaining $14 \%$. Nineteen participants were excluded because of incomplete data, leaving 275 participants for statistical analysis.

Mean \pm SD age was $35.8 \pm 13.0$ years, $75 \%$ of participants were women, close to two thirds of surveyed adults were born in the state of Oaxaca and, on average, had lived in San Quintin for almost two decades (Table 1). Number of completed school years was significantly lower among women $(p=0.004)$, and almost $82 \%$ of the study population was literate, with a significant sex difference $(p=0.016)$. Although the majority of women were homemakers, nearly $40 \%$ worked as field laborers. Sixty percent of study participants self-identified as having an indigenous ethnic background.

Seven percent of the population reported consuming alcohol 2-3 times per month, with beer being the most frequently consumed alcoholic beverage. Only $8 \%$ of participants were current smokers, significantly higher in 
Table 1 Socio-demographic, lifestyle, clinical, and laboratory characteristics, stratified by sex, among adults in San Quintin, Baja California, Mexico - 2013-2014 ( $n=275)$

\begin{tabular}{|c|c|c|c|c|}
\hline Characteristics $^{a}$ & $\begin{array}{l}\text { Total } \\
(n=275)\end{array}$ & $\begin{array}{l}\text { Men } \\
(n=70)\end{array}$ & $\begin{array}{l}\text { Women } \\
(n=205)\end{array}$ & $p$-value* \\
\hline \multicolumn{5}{|l|}{ Socio-demographic } \\
\hline Age (years) & $35.8 \pm 13.0$ & $37.0 \pm 13.6$ & $35.4 \pm 12.8$ & 0.367 \\
\hline Birth state, (\%) & & & & 0.704 \\
\hline Born in Oaxaca & $178(64.7)$ & $48(68.6)$ & $130(63.4)$ & \\
\hline Born in Baja California & $47(17.1)$ & $10(14.3)$ & $37(18.1)$ & \\
\hline Other & $50(18.2)$ & $12(17.1)$ & $38(18.5)$ & \\
\hline Income ${ }^{b}$ (pesos, monthly), (\%) & & & & 0.887 \\
\hline $0-2399$ & $55(20.0)$ & $12(17.1)$ & $43(21.0)$ & \\
\hline 2400-3199 & $69(25.1)$ & $17(24.3)$ & $52(25.4)$ & \\
\hline $3200-4799$ & $73(26.5)$ & $20(28.6)$ & $53(25.9)$ & \\
\hline $4800-18,000$ & $78(28.4)$ & $21(30.0)$ & $57(27.8)$ & \\
\hline Finished school ${ }^{c}$ (years) & $4.8 \pm 4.0$ & $6.0 \pm 4.0$ & $4.4 \pm 3.9$ & 0.004 \\
\hline Ethnic group, (\%) & & & & 0.673 \\
\hline Mestizo & $108(39.3)$ & $26(37.1)$ & $82(40.0)$ & \\
\hline Indigenous & $167(60.7)$ & $44(62.9)$ & $123(60.0)$ & \\
\hline Indigenous group ${ }^{c},(\%)$ & & & & 0.583 \\
\hline Mixtec & $95(62.1)$ & $24(58.5)$ & $71(63.4)$ & \\
\hline Other & $58(37.9)$ & $17(41.5)$ & $41(36.6)$ & \\
\hline Literate, (\%) & & & & 0.016 \\
\hline Yes & $225(81.8)$ & $64(91.4)$ & $161(78.5)$ & \\
\hline No & $50(18.2)$ & $6(8.6)$ & $44(21.5)$ & \\
\hline Speaks indigenous language ${ }^{c},(\%)$ & & & & 0.312 \\
\hline Yes & $107(39.2)$ & $31(44.3)$ & $76(36.4)$ & \\
\hline No & $166(60.8)$ & $39(55.7)$ & $127(62.6)$ & \\
\hline Occupation, (\%) & & & & $<0.0001$ \\
\hline Field laborer & $130(47.3)$ & $49(70.0)$ & $81(39.5)$ & \\
\hline Home maker & $107(38.9)$ & $3(4.3)$ & $104(50.7)$ & \\
\hline Other & $38(13.8)$ & $18(25.7)$ & $20(9.8)$ & \\
\hline Residence in San Quintin (years) & $18.1 \pm 8.5$ & $18.1 \pm 9.4$ & $18.1 \pm 8.1$ & 0.949 \\
\hline \multicolumn{5}{|l|}{ Lifestyle } \\
\hline Current smoking status, (\%) & & & & $<0.0001$ \\
\hline Yes & $21(7.6)$ & $16(22.9)$ & $5(2.4)$ & \\
\hline No & $254(92.4)$ & $54(77.1)$ & $200(97.6)$ & \\
\hline Alcohol intake, (\%) & & & & $<0.0001$ \\
\hline Occasionally or more & $31(11.3)$ & $21(30.0)$ & $10(4.9)$ & \\
\hline Rarely or never & $244(88.7)$ & $49(70.0)$ & $195(95.1)$ & \\
\hline Total activity score & $9.0 \pm 2.6$ & $9.1 \pm 2.9$ & $9.0 \pm 2.5$ & 0.667 \\
\hline \multicolumn{5}{|l|}{ Clinical } \\
\hline Weight (kg) & $65.0 \pm 12.7$ & $69.0 \pm 13.4$ & $63.6 \pm 12.2$ & $<0.0001$ \\
\hline Height (cm) & $150.8 \pm 8.9$ & $159.5 \pm 6.1$ & $147.8 \pm 7.6$ & $<0.0001$ \\
\hline Body mass index $\left(\mathrm{kg} / \mathrm{m}^{2}\right)$ & $28.7 \pm 5.6$ & $27.1 \pm 4.9$ & $29.2 \pm 5.8$ & 0.006 \\
\hline
\end{tabular}


Table 1 Socio-demographic, lifestyle, clinical, and laboratory characteristics, stratified by sex, among adults in San Quintin, Baja California, Mexico - 2013-2014 ( $n=275)$ (Continued)

\begin{tabular}{|c|c|c|c|c|}
\hline Characteristics $^{a}$ & $\begin{array}{l}\text { Total } \\
(n=275)\end{array}$ & $\begin{array}{l}\text { Men } \\
(n=70)\end{array}$ & $\begin{array}{l}\text { Women } \\
(n=205)\end{array}$ & $p$-value \\
\hline Waist circumference $(\mathrm{cm})$ & $96.1 \pm 12.6$ & $91.8 \pm 10.4$ & $97.6 \pm 12.9$ & $<0.0001$ \\
\hline Blood pressure, systolic (mmHg) & $123.7 \pm 18.8$ & $131.2 \pm 15.3$ & $121.1 \pm 19.3$ & $<0.001$ \\
\hline Blood pressure, diastolic $(\mathrm{mmHg})$ & $78.8 \pm 10.4$ & $81.4 \pm 7.7$ & $77.9 \pm 11.1$ & 0.005 \\
\hline \multicolumn{5}{|l|}{ Laboratory } \\
\hline Total cholesterol (mmol/L) & $4.1 \pm 0.9$ & $4.0 \pm 1.0$ & $4.1 \pm 0.9$ & 0.437 \\
\hline HDL cholesterol (mmol/L) & $1.1 \pm 0.3$ & $1.0 \pm 0.3$ & $1.1 \pm 0.3$ & 0.002 \\
\hline LDL cholesterol (mmol/L) & $2.1 \pm 0.7$ & $2.2 \pm 0.8$ & $2.1 \pm 0.6$ & 0.650 \\
\hline Triglycerides (mmol/L) & $2.0 \pm 1.1$ & $2.1 \pm 1.1$ & $1.9 \pm 1.0$ & 0.169 \\
\hline Glycated hemoglobin (A1c, \%) & $6.1 \pm 1.4$ & $6.1 \pm 1.4$ & $6.2 \pm 1.5$ & 0.524 \\
\hline
\end{tabular}

Abbreviations: $\mathrm{kg}$ kilograms, $\mathrm{cm}$ centimeters, $\mathrm{mmHg}$ millimeters of mercury, $\mathrm{mmol} / \mathrm{L}$ millimoles per liter

${ }^{a}$ Continuous variables are reported as mean \pm standard deviation. Categorical variables are reported as number of participants and percentage in parenthesis

${ }^{\mathrm{b}}$ Official currency of Mexico: Peso Mexicano

${ }^{\mathrm{C}}$ Due to missing values, not all frequencies add up to $100 \%$. Missing observations: Finished school $n=8$; Indigenous group $n=14$; Speaks indigenous

language $n=2$

*T-test or Chi Square Test for $p$ values. Significant values $(p<0.05)$ are shown in bold text

men $(22.9 \%)$ compared to women $(2.4 \%)(p<0.001)$. The mean \pm SD physical activity score was $9.0 \pm 2.6$ and did not differ by sex.

Mean $\pm \mathrm{SD}$ BMI was $28.7 \pm 5.6 \mathrm{~kg} / \mathrm{m}^{2}$, however, women had a significantly higher BMI compared to men $(p=0.006)$. Obesity (BMI $\left.\geq 30 \mathrm{~kg} / \mathrm{m}^{2}\right)$ was observed in $25.7 \%$ of men and $40.5 \%$ of women. There was no sex difference by total and LDL-cholesterol, and triglyceride levels, yet men had significantly lower HDL-cholesterol $(p=0.002)$. The mean \pm SD HbA1c for the sample was $6.1 \pm 1.4 \%$, with no sex difference.

Prevalence of Type $2 \mathrm{DM}$ and MetS was 21.8 and 53.1\%, respectively (Table 2). Both conditions were higher in women; however, the difference by sex was only statistically significant for MetS $(p=0.011)$. Participants self-identified as having an indigenous background had a 19 and 53\% prevalence of Type $2 \mathrm{DM}$ and MetS, respectively; yet, there was no significant difference with those who identified as being mestizo. A large waist circumference was found in $80.7 \%$ of the population, significantly higher in women $(p<$ 0.001 ), where more than $90 \%$ of adult women had a waist girth $\geq 80 \mathrm{~cm}$. Eight percent of adults had hypertension and $28.7 \%$ had normal-high blood pressure, and more than half of adults displayed dyslipidemia.

In bivariate analyses, age, sex, schooling, and literacy were associated with Type 2 DM (Table 3); while age, sex, schooling, literacy and birth state were associated with MetS (Table 4).

After controlling for age, those who completed at least elementary school were almost $60 \%$ less likely to have Type $2 \mathrm{DM}$ (adjusted OR $[\mathrm{AOR}]=0.39,95 \% \mathrm{CI}$ $0.20,0.77$ ) (Table 5). Women had 2.27 (95\% CI: 1.23, 4.14) higher odds of MetS compared to men, after adjusting for age and education. Furthermore, after controlling for sex and age, completion of at least elementary school was inversely associated with MetS ( $\mathrm{AOR}=0.62,95 \% \mathrm{CI} 0.37,0.94)$.

Table 2 Type 2 Diabetes Mellitus, Metabolic Syndrome, and criteria diagnosis, stratified by sex, among adults in San Quintin, Baja California, Mexico - 2013-2014 $(n=275)$

\begin{tabular}{lllll}
\hline Diagnosis $^{\mathrm{a}}$ & $\begin{array}{l}\text { Total } \\
(n=275)\end{array}$ & $\begin{array}{l}\text { Men } \\
(n=70)\end{array}$ & $\begin{array}{l}\text { Women } \\
(n=205)\end{array}$ & $p$-value $^{*}$ \\
\hline Type 2 DM $^{\mathrm{b}}$ & $60(21.8)$ & $13(18.6)$ & $47(22.9)$ & 0.446 \\
Metabolic Syndrome $^{c}$ & $146(53.1)$ & $28(40.0)$ & $118(57.6)$ & $\mathbf{0 . 0 1 1}$ \\
Large waist circumference $^{\mathrm{d}}$ & $222(80.7)$ & $36(51.4)$ & $186(90.7)$ & $<\mathbf{0 . 0 0 1}$ \\
Normal-high blood pressure $^{\mathrm{e}}$ & $79(28.7)$ & $25(35.7)$ & $54(26.3)$ & 0.135 \\
Hypertension $^{f}$ & $22(8.0)$ & $6(8.6)$ & $16(7.8)$ & 0.838 \\
Hypertriglyceridemia $^{\mathrm{g}}$ & $144(52.4)$ & $39(55.7)$ & $105(51.2)$ & 0.516 \\
Low HDL cholesterol $^{\mathrm{h}}$ & $187(68.0)$ & $42(60.0)$ & $145(70.7)$ & 0.097 \\
\hline
\end{tabular}

Abbreviations: Type 2 DM Type 2 Diabetes Mellitus, HDL high-density lipoprotein ${ }^{a} n(\%)$

${ }^{\mathrm{b}}$ Type 2 Diabetes Mellitus was defined as a glycated hemoglobin A1c $\geq 6.5 \%$, according to the American Diabetes Association criteria

${ }^{c}$ Metabolic Syndrome was defined following the International Diabetes Federation criteria: Ethnic-specific central obesity as waist circumference $\geq 90$ $\mathrm{cm}$ in men and $\geq 80 \mathrm{~cm}$ in women for this population, plus any two of the following: elevated triglycerides $\geq 1.7 \mathrm{mmol} / \mathrm{L}$; reduced $\mathrm{HDL}$-cholesterol $<1.03$ $\mathrm{mmol} / \mathrm{L}$ in men and $<1.20 \mathrm{mmol} / \mathrm{L}$ in women; elevated blood pressure as systolic blood pressure $\geq 130 \mathrm{mmHg}$ or diastolic blood pressure $85 \geq \mathrm{mmHg}$; and previously diagnosed with Type 2 diabetes mellitus

${ }^{d}$ Large waist circumference was defined as $\geq 90 \mathrm{~cm}$ in men and $\geq 80 \mathrm{~cm}$ in women eNormal-high blood pressure was defined as systolic blood pressure 130-139 $\mathrm{mmHg}$ or diastolic blood pressure $85-89 \mathrm{mmHg}$, according to the Norma Oficial Mexicana criteria

${ }^{f}$ Hypertension was defined as having a blood pressure $\geq 140 / 90 \mathrm{mmHg}$, according to the Norma Oficial Mexicana criteria

${ }^{9}$ Hypertriglyceridemia was defined as triglycerides $\geq 1.7 \mathrm{mmol} / \mathrm{L}$

h Low HDL cholesterol was defined as HDL cholesterol $<1.03 \mathrm{mmol} / \mathrm{L}$ in men and $<1.20 \mathrm{mmol} / \mathrm{L}$ in women

*Chi square test for $p$ values. Significant values $(p<0.05)$ are shown in bold text 
Table 3 Socio-demographic and lifestyle characteristics by Type 2 Diabetes Mellitus ${ }^{\mathrm{a}}$ diagnosis among adults in San Quintin, Baja California, Mexico - 2013-2014 ( $n=275)$

\begin{tabular}{|c|c|c|c|c|}
\hline Characteristic & $\begin{array}{l}\text { Type 2 DM } \\
(n=60)\end{array}$ & $\begin{array}{l}\text { No Type } 2 \text { DM } \\
(n=215)\end{array}$ & OR $(95 \% \mathrm{Cl})$ & $p$-value \\
\hline \multicolumn{5}{|l|}{ Socio-demographic } \\
\hline \multicolumn{5}{|l|}{ Sex, (\%) } \\
\hline Male & $13(21.7)$ & $57(26.5)$ & 1.00 & \\
\hline Female & $47(78.3)$ & $158(73.5)$ & $1.30(0.66,2.59)$ & 0.446 \\
\hline Age (years) & $43.5 \pm 14.6$ & $33.6 \pm 11.6$ & $1.06(1.03,1.08)$ & $<0.0001$ \\
\hline Birth state, (\%) & & & & 0.130 \\
\hline Other & $15(25.0)$ & $35(16.3)$ & 1.00 & \\
\hline Born in Oaxaca & $39(65.0)$ & $139(64.7)$ & $0.66(0.33,1.32)$ & 0.725 \\
\hline Born in Baja California & $6(10.0)$ & $41(19.0)$ & $0.34(0.12,0.99)$ & 0.068 \\
\hline \multicolumn{5}{|l|}{ Income ${ }^{b}$ (pesos, monthly), (\%) } \\
\hline$<3200$ & $30(50.0)$ & $94(43.7)$ & 1.00 & \\
\hline$\geq 3200$ & $30(50.0)$ & $121(56.3)$ & $0.78(0.44,1.39)$ & 0.387 \\
\hline \multicolumn{5}{|l|}{ Finished schoolc, (\%) } \\
\hline$<6$ y (Less than Elementary) & $46(76.7)$ & $102(47.4)$ & 1.00 & \\
\hline$\geq 6$ y (Elementary or More) & $14(23.3)$ & $113(52.6)$ & $0.27(0.14,0.53)$ & $<0.001$ \\
\hline \multicolumn{5}{|l|}{ Ethnic group, (\%) } \\
\hline Mestizo & $28(46.7)$ & $80(37.2)$ & 1.00 & \\
\hline Indigenous & $32(53.3)$ & $135(62.8)$ & $0.68(0.38,1.21)$ & 0.185 \\
\hline \multicolumn{5}{|l|}{ Indigenous groupc, (\%) } \\
\hline Other & $11(35.5)$ & $47(38.5)$ & 1.00 & \\
\hline Mixteco & $20(64.5)$ & $75(61.5)$ & $1.14(0.50,2.59)$ & 0.755 \\
\hline \multicolumn{5}{|l|}{ Literate, (\%) } \\
\hline No & $20(33.3)$ & $30(14.0)$ & 1.00 & \\
\hline Yes & $40(66.7)$ & $185(86.0)$ & $0.32(0.17,0.63)$ & 0.001 \\
\hline \multicolumn{5}{|l|}{ Speaks in indigenous language ${ }^{c},(\%)$} \\
\hline No & $37(63.8)$ & $129(60.0)$ & 1.00 & \\
\hline Yes & $21(36.2)$ & $86(40.0)$ & $0.85(0.47,1.55)$ & 0.600 \\
\hline Occupation, (\%) & & & & 0.360 \\
\hline Other & $8(13.3)$ & $30(14.0)$ & 1.00 & \\
\hline Field laborer & $24(40.0)$ & $106(49.3)$ & $0.85(0.35,2.08)$ & 0.340 \\
\hline Home maker & $28(46.7)$ & $79(36.7)$ & $1.33(0.55,3.24)$ & 0.248 \\
\hline Residence in San Quintin (years) & $19.9 \pm 8.3$ & $17.6 \pm 8.5$ & $1.03(0.99,1.07)$ & 0.080 \\
\hline \multicolumn{5}{|l|}{ Lifestyle } \\
\hline \multicolumn{5}{|l|}{ Smoking status, (\%) } \\
\hline No & $53(88.3)$ & $201(93.5)$ & 1.00 & \\
\hline Yes & $7(11.7)$ & $14(6.5)$ & $1.90(0.73,4.93)$ & 0.180 \\
\hline
\end{tabular}


Table 3 Socio-demographic and lifestyle characteristics by Type 2 Diabetes Mellitus ${ }^{\mathrm{a}}$ diagnosis among adults in San Quintin, Baja California, Mexico - 2013-2014 ( $n=275)$ (Continued)

\begin{tabular}{lllll}
\hline Characteristic & $\begin{array}{l}\text { Type 2 DM } \\
(n=60)\end{array}$ & $\begin{array}{l}\text { No Type 2 DM } \\
(n=215)\end{array}$ & OR (95\% Cl) & $p$-value \\
\hline Alcohol intake ${ }^{\text {d }}(\%)$ & & $191(88.8)$ & 1.00 & $1.05(0.43,2.57)$ \\
$\quad$ Rarely or never & $53(88.3)$ & $24(11.2)$ & $1.03(0.93,1.15)$ & 0.913 \\
$\quad$ Occasionally or more & $7(11.7)$ & $9.0 \pm 2.6$ & 0.561 \\
Total activity score & $9.2 \pm 2.8$ & &
\end{tabular}

Abbreviations: Type 2 DM Type 2 Diabetes Mellitus, OR odds ratio, $\mathrm{Cl}$ confidence interval, $y$ years

${ }^{\text {aType }} 2$ Diabetes Mellitus was defined as a glycated hemoglobin A1c $\geq 6.5 \%$, according to the American Diabetes Association criteria

${ }^{b}$ Official currency of Mexico: Peso Mexicano

'Due to missing values, not all frequencies add up to $100 \%$. Missing observations: School years $n=8$; Indigenous group $n=14$; Speaks in indigenous

language $\mathrm{n}=2$

${ }^{d}$ Alcohol intake defined as 'rarely or never' includes responses: never/rarely and 2-3 times per month; 'occasionally or more' includes responses: 1-2 times per week and 3 times or more per week

*Chi square test and logistic regression used for $p$ values. Significant values $(p<0.05)$ are shown in bold text

Table 4 Socio-demographic and lifestyle characteristics by Metabolic Syndrome ${ }^{\text {a }}$ diagnosis among adults in San Quintin, Baja California, Mexico - 2013-2014 ( $n=275)$

\begin{tabular}{|c|c|c|c|c|}
\hline Characteristic & $\begin{array}{l}\text { MetS } \\
(n=146)\end{array}$ & $\begin{array}{l}\text { No MetS } \\
(n=129)\end{array}$ & OR $(95 \% \mathrm{Cl})$ & $p$-value ${ }^{*}$ \\
\hline \multicolumn{5}{|l|}{ Socio-demographic } \\
\hline \multicolumn{5}{|l|}{ Sex, $(\%)$} \\
\hline Male & $28(19.2)$ & $42(32.6)$ & 1.00 & \\
\hline Female & $118(80.8)$ & $87(67.4)$ & $2.03(1.17,3.54)$ & 0.011 \\
\hline Age (years) & $39.6 \pm 13.3$ & $31.4 \pm 11.2$ & $1.01(1.04,1.08)$ & $<0.0001$ \\
\hline Birth state, (\%) & & & & 0.017 \\
\hline Other & $30(20.5)$ & $20(15.5)$ & 1.00 & \\
\hline Born in Oaxaca & $100(68.5)$ & $78(60.5)$ & $0.86(0.45,1.62)$ & 0.147 \\
\hline Born in Baja California & $16(11.0)$ & $31(24.0)$ & $0.34(0.15,0.79)$ & 0.005 \\
\hline \multicolumn{5}{|l|}{ Income ${ }^{\mathrm{b}}$ (pesos, monthly), (\%) } \\
\hline$<3200$ & $68(46.6)$ & $56(43.4)$ & 1.00 & \\
\hline$\geq 3200$ & $78(53.4)$ & $73(56.6)$ & $0.88(0.55,1.42)$ & 0.599 \\
\hline \multicolumn{5}{|l|}{ Finished schoolc, (\%) } \\
\hline$<6$ y (Less than Elementary) & $94(64.4)$ & $53(41.9)$ & 1.00 & \\
\hline$\geq 6$ y (Elementary or More) & $52(35.6)$ & $75(58.1)$ & $0.40(0.24,0.65)$ & 0.0002 \\
\hline \multicolumn{5}{|l|}{ Ethnic group, (\%) } \\
\hline Mestizo & $58(39.7)$ & $50(38.8)$ & 1.00 & \\
\hline Indigenous & $88(60.3)$ & $79(61.2)$ & $0.96(0.59,1.56)$ & 0.870 \\
\hline \multicolumn{5}{|l|}{ Indigenous groupc, (\%) } \\
\hline Other & $33(38.4)$ & $25(37.3)$ & 1.00 & \\
\hline Mixteco & $53(61.6)$ & $42(62.7)$ & $0.96(0.50,1.85)$ & 0.894 \\
\hline \multicolumn{5}{|l|}{ Literate, (\%) } \\
\hline No & $35(24.0)$ & $15(11.6)$ & 1.00 & \\
\hline Yes & $111(76.0)$ & $114(88.4)$ & $0.42(0.22,0.81)$ & 0.008 \\
\hline \multicolumn{5}{|c|}{ Speaks in indigenous language ${ }^{c},(\%)$} \\
\hline No & $84(58.3)$ & $82(63.6)$ & 1.00 & \\
\hline Yes & $60(41.7)$ & $47(36.4)$ & $1.25(0.77,2.03)$ & 0.377 \\
\hline
\end{tabular}


Table 4 Socio-demographic and lifestyle characteristics by Metabolic Syndrome ${ }^{a}$ diagnosis among adults in San Quintin, Baja California, Mexico - 2013-2014 ( $n=275)$ (Continued)

\begin{tabular}{lllll}
\hline Characteristic & $\begin{array}{l}\text { MetS } \\
(n=146)\end{array}$ & $\begin{array}{l}\text { No MetS } \\
(n=129)\end{array}$ & OR (95\% Cl) & 0.998 \\
\hline $\begin{array}{l}\text { Occupation, (\%) } \\
\quad \text { Other }\end{array}$ & $20(13.7)$ & $18(14.0)$ & 1.00 & \\
$\quad$ Field laborer & $69(47.3)$ & $61(47.3)$ & $1.02(0.49,2.10)$ & 0.984 \\
$\quad$ Home maker & $57(39.0)$ & $50(38.7)$ & $1.03(0.48,2.23)$ & 0.950 \\
Residence in San Quintin (years) & $18.1 \pm 8.3$ & $18.1 \pm 8.6$ & $1.00(0.97,1.03)$ & 0.984
\end{tabular}

Lifestyle

Smoking status, (\%)

No $135(92.5)$

$119(92.2)$

1.00

Yes

$11(7.5)$

$10(7.8)$

$0.97(0.40,2.37)$

Alcohol intake ${ }^{\mathrm{d}},(\%)$

Rarely or never

1.00

Occasionally or more

Abbreviations: MetS Metabolic Syndrome, OR odds ratio, $\mathrm{Cl}$ confidence interval, $y$ years

${ }^{a}$ Metabolic Syndrome was defined following the International Diabetes Federation criteria: Ethnic-specific central obesity as waist circumference $\geq 90 \mathrm{~cm}$ in men and $\geq 80 \mathrm{~cm}$ in women for this population, plus any two of the following: elevated triglycerides $\geq 1.7 \mathrm{mmol} / \mathrm{L}$; reduced $\mathrm{HDL}$-cholesterol $<1.03 \mathrm{mmol} / \mathrm{L}$ in men and $<1.20 \mathrm{mmol} / \mathrm{L}$ in women; elevated blood pressure as systolic blood pressure $\geq 130 \mathrm{mmHg}$ or diastolic blood pressure $\geq 85 \mathrm{mmHg}$; and previously diagnosed Type 2 Diabetes Mellitus

${ }^{b}$ Official currency of Mexico: Peso Mexicano

'Due to missing values, not all frequencies add up to $100 \%$. Missing observations: School years $n=8$; Indigenous group $n=14$; Speaks in indigenous language $\mathrm{n}=2$

dAlcohol intake defined as 'rarely or never' includes responses: never/rarely and 2-3 times per month; 'occasionally or more' includes responses: 1-2 times per week and 3 times or more per week

${ }^{*}$ Chi square test used for $p$ values. Significant values $(p<0.05)$ are shown in bold text

Table 5 Multivariable logistic regression ${ }^{\mathrm{a}}$ analyses of Type 2 Diabetes Mellitus ${ }^{\mathrm{b}}$ and Metabolic Syndrome ${ }^{\mathrm{c}}$ in adults in San Quintin, Baja California, Mexico - 2013-2014 ( $n=275)$

\begin{tabular}{|c|c|c|c|c|c|c|c|c|}
\hline \multirow[b]{3}{*}{ Characteristic } & \multicolumn{4}{|c|}{ Type 2 Diabetes Mellitus ${ }^{b}$} & \multicolumn{4}{|c|}{ Metabolic Syndrome $^{c}$} \\
\hline & \multicolumn{2}{|l|}{ Full Model } & \multicolumn{2}{|l|}{ Final Model } & \multicolumn{2}{|l|}{ Full Model } & \multicolumn{2}{|l|}{ Final Model } \\
\hline & OR $(95 \% \mathrm{Cl})$ & $p$-value ${ }^{*}$ & OR $(95 \% \mathrm{Cl})$ & $p$-value & OR (95\% Cl) & $p$-value & OR $(95 \% \mathrm{Cl})$ & $p$-value \\
\hline Age & $1.05(1.02,1.08)$ & 0.0002 & $1.05(1.03,1.07)$ & $<0.0001$ & $1.06(1.03,1.09)$ & $<0.0001$ & $1.06(1.03,1.08)$ & $<0.0001$ \\
\hline Women $^{\mathrm{d}}$ & $1.25(0.59,2.66)$ & 0.567 & - & - & $2.27(1.23,4.20)$ & 0.009 & $2.27(1.23,4.14)$ & 0.009 \\
\hline $\begin{array}{l}\geq 6 \text { y of schooling } \\
\text { (Elementary) }\end{array}$ & $0.42(0.20,0.88)$ & 0.005 & $0.39(0.20,0.77)$ & 0.007 & $0.59(0.33,1.00)$ & 0.051 & $0.62(0.37,0.94)$ & 0.050 \\
\hline Literate $^{f}$ & $0.84(0.38,1.85)$ & 0.665 & - & - & $1.11(0.50,2.42)$ & 0.798 & - & - \\
\hline Born in Oaxaca ${ }^{g}$ & N/A & & & & $0.62(0.31,1.25)$ & 0.385 & - & - \\
\hline \multirow[t]{2}{*}{ Born in $\mathrm{BC}^{\mathrm{g}}$} & N/A & & & & $0.65(0.26,1.62)$ & 0.644 & - & - \\
\hline & & & GOF & 0.801 & & & GOF & 0.323 \\
\hline
\end{tabular}

Abbreviations: $y$ years, $B C$ Baja California, OR odds ratio, $C l$ confidence interval, GOF Goodness of Fit Test, -- not included in the final model

a Logistic regression modeling, presenting odds ratio and $95 \%$ confidence interval

${ }^{\mathrm{b}}$ Type 2 Diabetes Mellitus was defined as a glycated hemoglobin A1c $\geq 6.5 \%$, according to the American Diabetes Association criteria

${ }^{C}$ Metabolic Syndrome was defined following the International Diabetes Federation criteria: Ethnic-specific central obesity as waist circumference $\geq 90 \mathrm{~cm}$ in men and $\geq 80 \mathrm{~cm}$ in women for this population, plus any two of the following: elevated triglycerides $\geq 1.7 \mathrm{mmol} / \mathrm{L}$; reduced $\mathrm{HDL}$-cholesterol $<1.03 \mathrm{mmol} / \mathrm{L}$ in $\mathrm{men}$ and $<1.20 \mathrm{mmol} / \mathrm{L}$ in women; elevated blood pressure as systolic blood pressure $\geq 130 \mathrm{mmHg}$ or diastolic blood pressure $\geq 85 \mathrm{mmHg}$; and previously diagnosed Type 2 Diabetes Mellitus

${ }^{\mathrm{d}}$ Reference: Men

Reference: $<6$ y of schooling (No Elementary)

fReference: Illiterate

9Reference: Other

${ }^{*} P$ values calculated by logistic regression modeling, adjusted for all other listed variables in each of the models (full and final). Significant values $(p<0.05)$ are shown in bold text 


\section{Discussion}

This study found a Type $2 \mathrm{DM}$ and MetS prevalence in adults in a rural indigenous community in San Quintin, Mexico, of 21.8 and $53.1 \%$, respectively, with a higher prevalence of both conditions in women.

Prevalence of Type 2 DM in the community was high. The latest national survey results found a diabetes prevalence of $9.6 \%$ in northern rural areas of Mexico [23], much lower than our results. Escobedo et al. and Alvarado-Osuna et al., determined diabetes prevalence in indigenous groups in Southern Mexico, with our study population showing a much higher prevalence than that observed in Zapotec (8.7\%), Mixe (6.9\%) [16] and Otomi (4.4\%) [15] groups. Zapotec and Mixe women had a higher diabetes prevalence, 13.3 and $7.1 \%$, respectively, when compared to Zapotec and Mixe men, with a 6.2 and $5.7 \%$ prevalence, respectively. Our study shared the commonality of a higher proportion of women (22.9\%) with Type 2 DM, in contrast to men (18.6\%), yet recognize the larger prevalence of the condition in our sample of rural indigenous adults. Compared to the findings reported by Goodman et al., in the same community eight years prior (26.2\%), the prevalence of Type 2 DM was reduced by $16.8 \%$ [17]. Goodman et al., defined Type $2 \mathrm{DM}$ as having a $\mathrm{HbA} 1 \mathrm{c}$ of $\geq 6.5 \%$ or a fasting glucose of $\geq 126 \mathrm{mg} / \mathrm{dL}$; it was unclear of the proportion of those defined by either HbA1c or fasting glucose.

Similarly, we found a very high MetS prevalence. MetS was $29 \%$ higher than what was previously found in the same community in 2008 (41.1\%) [17]. We are cautious when making direct comparisons because the previous study followed the ATP III guideline criteria, while we used the IDF definition, in which central obesity, using ethnic-specific waist circumference cut-offs, is a requirement. Our findings were similar to the ENSANUT 2006 MetS prevalence of $49.8 \%$ [12], and comparable to a recent Northeastern Mexico study that reported an overall MetS prevalence of $54.8 \%$ in the state of Nuevo Leon [24], with closer resemblance to the IDF definition. Although this Northeastern Mexico study included an older population (mean \pm SD age $51.0 \pm 19.0$ and $50.7 \pm$ 19.0 years, men and women, respectively), teenagers were also included. Considering the MetS prevalence in Latin America, our findings sit outside the estimated range of $18.8-43.4 \%$ [11].

A higher prevalence of MetS was observed in women when compared to men (56.7\% vs $40.0 \%$ ), analogous to the Northeastern Mexico study findings $(60.4 \%$ in women vs $48.9 \%$ in men) [24]. This was further observed in our multivariable model results, when after adjusting for covariates, women had a 2.27 (95\% CI 1.23, 4.14) greater odds of MetS compared to men. The 40\% MetS prevalence in men was akin to the general prevalence reported by Ramirez-Vargas et al., in a sample of Oaxacan men living in rural and urban communities (40.0\% in rural vs $41.2 \%$ in urban) [19]; yet our results were $31 \%$ higher than the prevalence of MetS among those Oaxacan men living in a rural area (27.6\%). Regardless of the differences between studies, our results indicate that the prevalence of MetS was high in our study population and has increased since the last cross-sectional examination.

Schooling was independently associated with both Type 2 DM $(\mathrm{AOR}=0.39,95 \%$ CI 0.20, 0.77) and MetS $(\mathrm{AOR}=0.62,95 \%$ CI 0.37, 0.94). Recent Type 2 DM epidemiological surveillance data highlighted that among all identified cases for the 2016 year, almost 50\% had less than elementary school education, with less cases having had at least elementary school [25]. The ENSANUT 2006 national results reported that the lower the education level, the higher prevalence of MetS [26]. Those adults with less than elementary school education had the highest prevalence of MetS, 60.1\% (95\% CI 56.9-63.3\%) while those with more than high school attainment had 39.3\% (95\% CI 32.5-46.6\%) prevalence of MetS in 2006. This evidence supports our findings, underscoring educational level as an important socio-demographic characteristic and possible protective factor for Type $2 \mathrm{DM}$ and MetS in indigenous Mexican groups.

The population also exhibited altered cardiovascular characteristics. A large waist circumference was observed in a large proportion of the study population, indicating excess abdominal body fat and health consequences associated with central obesity. Intra-abdominal fat, MetS and Type 2 DM are inter-related [27], thus having an elevated intra-abdominal fat accumulation may have a direct function in the development of MetS [28] and Type 2 DM, because of its association with insulin resistance [27].

More than a third of participants were hypertensive or had normal-high blood pressure, worrisome since elevated blood pressure is a silent killer and major risk factor for CVD. The new hypertension guidelines acknowledge complications at lower levels of blood pressure, thus aggressive screening should be highlighted [29]. More than half of adults presented dyslipidemia, similar to adults in Northeastern Mexico, were two thirds had low HDL-cholesterol levels and almost half had elevated triglycerides [24].

The mean \pm SD total activity score for the population was $9.0 \pm 2.6$, with no sex difference. This translates to an average level of total activity based on transportation, television watching time, occupation-related intensity and recreational physical activity. We understand the relationship between physical activity and Type 2 DM and MetS, yet we did not see this in the present study.

We acknowledge the cross-sectional nature of the study design and related shortcomings, yet our study aim consisted of determining Type 2 DM and MetS prevalence 
while describing potential disease correlates in this particular population, without a singular exposure-disease relationship. Thereby, study limitations include self-reported lifestyle measures and incomplete information on family history, introducing information bias. Dietary intake was not assessed, and likelihood of residual confounding is recognised. Generalisability is restricted to neighboring communities with a demographically similar population.

Study strengths include the use of GIS mapping and systematic random sampling of households, which allowed us to obtain a more representative sample of the community than prior studies. The application of a detailed protocol performed by a rigorously trained team in a rural and inaccessible area is a notable attainment, while determining health outcomes with standardised and validated objective measures, applied in a global public health setting. Diabetes in on the rise in Mexico, thereby we consider our findings to be relevant and timely. We are contributing to the study of Mexican rural and indigenous communities, whilst providing crucial evidence to help inform interventions and health policy.

\section{Conclusions}

Findings elucidate the elements of Type $2 \mathrm{DM}$ and MetS in this rural population, while enhancing the literature on these two conditions and cardiometabolic risk factors in indigenous communities in Mexico. It is important to highlight that our study aimed to describe the magnitude of the burden of Type $2 \mathrm{DM}$ and MetS, the latter serving as an indicator of the number of additional community members at risk of Type 2 DM. Comprehensive community-inclusionary interventions should be pursued to help prevent and mitigate potential health consequences related to these conditions, especially including women. Education must be considered as a key factor when addressing the prevention of Type $2 \mathrm{DM}$ and MetS.

\footnotetext{
Abbreviations

95\% Cl: 95\% confidence interval; AOR: Adjusted odds ratio; ATP III: Adult treatment panel III; BMI: Body mass index; CVD: Cardiovascular disease; DBP: Diastolic blood pressure; ENSANUT: Encuesta Nacional de Salud y Nutrición (Spanish), Mexican National Health \& Nutrition Survey (English); HbA1c: Glycated hemoglobin; HDL-cholesterol: High density lipoprotein cholesterol; IDF: International diabetes federation; LDL-cholesterol: Low density lipoprotein cholesterol; MetS: Metabolic syndrome; SBP: Systolic blood pressure; SD: Standard deviation; SDSU: San Diego State University; Type 2 DM: Type 2 diabetes mellitus; UABC: Universidad Autonoma de Baja California; VIIDAl: Viaje Interinstitucional de Integración Docente, Asistencial y de Investigación (Spanish), Inter-institutional Field Experience for Integration, Teaching, Health Services and Research (English)
}

\section{Acknowledgements}

Kind regards to the residents of the San Quintin community for their trust and participation in the study. Special recognition to Professor Javier Martínez, school principal of Escuela Primaria Federal Bilingüe, for his incessant support and important role as key informant and liaison. We also wish to acknowledge the Old Mission Rotary Club and students and faculty from San Diego State University Graduate School of Public Health, University of California San Diego School of Medicine and the Universidad Autónoma de Baja California Facultad de Medicina y Psicología for their commitment and zealous work ethic.

\section{Funding}

Funding for the study, including design, data collection, logistics, training, and field work activities considering anthropometric instruments, point of care devices, and biochemical assays, was provided by the Fred $\mathrm{H}$. Bixby Foundation (Award C03093) and Universidad Autónoma de Baja California Facultad de Medicina y Psicología (Award 304/2/C/103/17), Tijuana, Baja California campus. Miss Pacheco is a trainee in the University of California San Diego Integrated Cardiovascular Epidemiology Fellowship (National Heart Lung and Blood Institute T32 HL079891-11). She was funded by the aforementioned traineeship as well as through the University of California San Diego School of Medicine Hispanic Center of Excellence (Health Resources and Services Administration D34HP31027).

\section{Availability of data and materials}

The datasets generated and/or analysed during the current study are not publicly available due to the internal policies of UABC' Facultad de Medicina and Psicología but are available from the corresponding author on reasonable request.

\section{Authors' contributions}

LSP led nutrition counseling, managed and analysed the collected data, interpreted results, and drafted the manuscript. DAHO assisted in field work logistics, contributed to power calculation, data collection, and statistical analysis in preparation for the manuscript. ElS helped develop the study design, led triage group coordination for field work data collection, and contributed to interpretation of results and discussion of manuscript. SB, San Diego State University team leader, helped conceive study and contributed to the interpretation of results and discussion, and acquired funding for project development. RSG, University of California San Diego team leader, participated in study design, interpretation of results and discussion. MS assisted in study logistics and triage group organization for data collection as well as manuscript discussion. MAF is the director of VIIDAI and Universidad Autónoma de Baja California team leader; he conceived, with SB, the study objective and design as well as implementation, led protocol design and research assistant trainings, contributed to discussion of manuscript, and acquired study funding. All authors read and approved the final manuscript.

\section{Ethics approval and consent to participate}

This study and all associated procedures were approved by the Bioethics Committee of the Facultad de Medicina y Psicología of UABC (No. 991/ 2013-2) and by the Institutional Review Board of SDSU's Human Research Protection Program (No. 1521089). In alignment with the tenets of the aforementioned institutional boards, written informed consent, in Spanish, was obtained from all study participants before study enrollment.

\section{Consent for publication}

Not applicable.

\section{Competing interests}

The authors declare that they have no competing interests.

\section{Publisher's Note}

Springer Nature remains neutral with regard to jurisdictional claims in published maps and institutional affiliations.

\section{Author details}

${ }^{1}$ Department of Family Medicine and Public Health, School of Medicine, University of California, San Diego, La Jolla, CA, USA. 'Facultad de Medicina y Psicología, Universidad Autónoma de Baja California, Tijuana, Baja California, Mexico. ${ }^{3}$ School of Public Health, San Diego State University, San Diego, CA, USA. ${ }^{4}$ Division of Infectious Diseases and Global Public Health, School of Medicine, University of California San Diego, La Jolla, CA, USA. 
Received: 23 June 2018 Accepted: 28 November 2018

Published online: 20 December 2018

\section{References}

1. Grundy SM. Metabolic syndrome update. Trends Cardiovasc Med. 2016; 26(4):364-73.

2. World Health Organization. Global report of diabetes [Internet]. Geneva: World Health Organization [cited 2018 May 2]. Available from: http://www. who.int/diabetes/global-report/en/

3. Grundy SM. Overnutrition, ectopic lipid and the metabolic syndrome. J Investig Med. 2016:64(6):1082-6.

4. Expert Panel on Detection, Evaluation, and Treatment of High Blood Cholesterol in Adults. Executive Summary of the Third Report of the National Cholesterol Education Program (NCEP) Expert Panel on Detection, Evaluation, and Treatment of High Blood Cholesterol in Adults (Adult Treatment Panel III). JAMA. 2001;285(19):2486-97.

5. Alberti KGMM, Zimmet PZ. Definition, diagnosis and classification of diabetes mellitus and its complications. Part 1: diagnosis and classification of diabetes mellitus. Provisional report of a WHO consultation. Diabet Med. 1998:15(7):539-53.

6. Alberti KGMM, Zimmet P, Shaw J. Metabolic syndrome-a new world-wide definition. A consensus statement from the international diabetes federation. Diabet Med. 2006;23(5):469-80.

7. Gami AS, Witt BJ, Howard DE, Erwin PJ, Gami LA, Somers VK, et al. Metabolic syndrome and risk of incident cardiovascular events and death. J Am Coll Cardiol. 2007:49(4):403-14.

8. Mottillo S, Filion KB, Genest J, Joseph L, Pilote L, Poirier P, et al. The metabolic syndrome and cardiovascular risk: a systematic review and metaanalysis. J Am Coll Cardiol. 2010;56(14):1113-32.

9. Grundy SM. Metabolic syndrome pandemic. Arterioscler Thromb Vasc Biol. 2008;28(4):629-36

10. Diabetes Atlas Eight Edition Committee. IDF diabetes atlas: 2017 Atlas [Internet]. Brussels: International Diabetes Federation [cited 2018 May 2]. Available from: http://www.diabetesatlas.org/resources/2017-atlas.html

11. Márquez-Sandoval F, Macedo-Ojeda G, Viramontes-Hörner D, Fernández Ballart J, Salas Salvadó J, Vizmanos B. The prevalence of metabolic syndrome in Latin America: a systematic review. Public Health Nutr. 2011; 14(10):1702-13.

12. Rojas R, Aguilar-Salinas CA, Jiménez-Corona A, Shamah-Levy T, Rauda J, AvilaBurgos $L$, et al. Metabolic syndrome in Mexican adults: results from the National Health and nutrition survey 2006. Salud Publica Mex. 2010:52(Suppl 1):S11-8.

13. Pan American Health Organization. Health in the Americas +, 2017 Edition. Regional outlook and country profiles [Internet]. Washington, D.C: Pan American Health Organization [cited 2018 Feb 3]. Available from: http://iris. paho.org/xmlui/handle/123456789/34321

14. Hernández-Ávila M, Rivera-Dommarco J, Shamah-Levy T, Cuevas-Nasu L, GómezAcosta LM, Gaona-Pineda EB, et al. Encuesta Nacional de Salud y Nutrición de Medio Camino 2016. ENSANUT MC 2016: Informe final de resultados 2016 [Internet]. Cuernavaca: Instituto Nacional de Salud Pública [cited 2018 March 3]. Available from: https://www.insp.mx/images/stories/2017/Avisos/docs/180315 encuesta_nacional_de_salud_y_nutricion_de_medio_Ca.pdf.

15. Alvarado-Osuna C, Milian-Suazo F, Valles-Sánchez V. Prevalencia de diabetes mellitus e hiperlipidemias en indígenas otomíes. Salud Publica Mex. 2001; 43(5):459-63.

16. Escobedo J, Chavira I, Martínez L, Velasco X, Escandón C, Cabral J. Diabetes and other glucose metabolism abnormalities in Mexican Zapotec and Mixe Indians. Diabet Med. 2010;27(4):412-6.

17. Goodman D, Fraga MA, Brodine S, Ibarra ML, Garfein RS. Prevalence of diabetes and metabolic syndrome in a migrant Mixtec population, Baja California. Mexico J Immigr Minor Heal. 2013;15(1):93-100.

18. Gobierno de Baja California. Programa de Infraestructura Básica para los Indígenas. Evaluación Específica de Desempeño 2013 [Internet]. Gobierno del Estado de Baja California [cited 2013 February 3]. Available from: http:// indicadores.bajacalifornia.gob.mx/Uploads/Difusion\%202013/ INDIGENAS\%20PIBAI\%20Contenido\%20Extenso.pdf.

19. Ramírez-Vargas E, Arnaud-Viñas M, Del R, Delisle H. Prevalence of the metabolic syndrome and associated lifestyles in adult males from Oaxaca, Mexico. Salud Publica Mex. 2007:49(2):94-102.

20. Centro de Investigación en Nutrición y Salud Instituto Nacional de Salud Pública. Manual de procedimientos para proyectos de nutrición [Internet]. Cuernavaca:
Instituto Nacional de Salud Pública [cited 2013 March 24]. Available from: http:// www.salud.gob.mx/unidades/cdi/documentos/proy nutricion.pdf.

21. Secretaría de Salud. Norma Oficial Mexicana NOM-030-SSA2-1999 para la prevención, tratamiento y control de la hipertensión arterial [Internet]. Mexico: Secretaría de Salud [cited 2013 May 15]. Available from: http:// www.salud.gob.mx/unidades/cdi/nom/030ssa29.html.

22. American Diabetes Association. Diagnosis and classification of diabetes mellitus. Diabetes Care 2010; 33 Suppl 1: S62-S69.

23. Gutiérrez JP, Rivera-Dommarco J, Shamah-Levy T, Villalpando-Hernández S, Franco A, Cuevas-Nasu L, et al. Encuesta Nacional de Salud y Nutrición 2012. ENSANUT 2012: Informe final de resultados 2012 [Internet]. Cuernavaca: Instituto Nacional de Salud Pública [cited 2018 April 28]. Available from: https://ensanut.insp.mx/informes/ENSANUT2012ResultadosNacionales.pdf.

24. Salas R, Bibiloni M del M, Ramos E, Villarreal JZ, Pons A, Tur JA, et al. Metabolic syndrome prevalence among Northern Mexican adult population. PLoS One 2014; 9(8): e105581.

25. Secretaría de Salud. Dirección General de Epidemiología. Sistema de vigilancia Hospitalaria de Diabetes Tipo 2. Boletín e cierre anual 2016 [Internet]. Mexico: Secretaría de Salud [cited 2018 April 17]. Available from: https://www.gob.mx/ cms/uploads/attachment/file/266507/dmt2_cierre_2016.pdf.

26. Olaiz-Fernández G, Rivera-Dommarco J, Shamah-Levy T, Rojas R, VillalpandoHernández S, Hernández-Avila M, et al. Encuesta Nacional de Salud y Nutrición 2006. ENSANUT 2006: Informe final de resultados 2006 [Internet]. Cuernavaca: Instituto Nacional de Salud Pública [cited 2018 April 7]. Available from: https://ensanut.insp.mx/informes/ensanut2006.pdf.

27. Han TS, Lean ME. A clinical perspective of obesity, metabolic syndrome and cardiovascular disease. JRSM Cardiovasc Dis. 2016;5:1-13.

28. Han TS, Williams K, Sattar N, Hunt KJ, Lean MEJ, Haffner SM. Analysis of obesity and hyperinsulinemia in the development of metabolic syndrome: San Antonio heart study. Obes Res. 2002;10(9):923-31.

29. 2017 ACC/AHA/AAPA/ABC/ACPM/AGS/APhA/ASH/ASPC/NMA/PCNA Guideline for the Prevention, Detection, Evaluation, and Management of High Blood Pressure in Adults: A Report of the American College of Cardiology/American Heart Association Task Force on Clinical Practice Guidelines. J Am Coll Cardiol 2018; 71(19): e127-e248.

Ready to submit your research? Choose BMC and benefit from:

- fast, convenient online submission

- thorough peer review by experienced researchers in your field

- rapid publication on acceptance

- support for research data, including large and complex data types

- gold Open Access which fosters wider collaboration and increased citations

- maximum visibility for your research: over $100 \mathrm{M}$ website views per year

At BMC, research is always in progress.

Learn more biomedcentral.com/submissions 